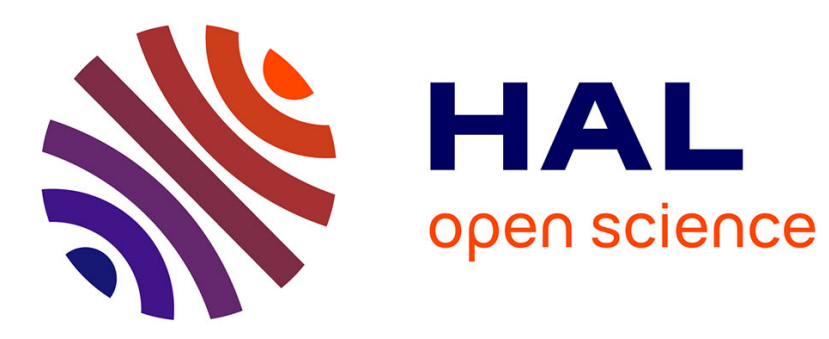

\title{
Réseaux agronomiques et échanges transfrontaliers franco-suisses au XIXe siècle \\ Fabien Knittel
}

\section{To cite this version:}

Fabien Knittel. Réseaux agronomiques et échanges transfrontaliers franco-suisses au XIXe siècle. Benjamin Castets Fontaine; Maxime Kaci; Jérôme Loiseau; Alexandre Moine. Deux frontières aux destins croisés ?, 36, Presses universitaires de Franche-Comté, pp.119-128, 2019, Les Cahiers de la MSHE Ledoux, 978-2-84867-644-9. hal-03248629

\section{HAL Id: hal-03248629 \\ https://univ-fcomte.hal.science/hal-03248629}

Submitted on 3 Jun 2021

HAL is a multi-disciplinary open access archive for the deposit and dissemination of scientific research documents, whether they are published or not. The documents may come from teaching and research institutions in France or abroad, or from public or private research centers.
L'archive ouverte pluridisciplinaire $\mathbf{H A L}$, est destinée au dépôt et à la diffusion de documents scientifiques de niveau recherche, publiés ou non, émanant des établissements d'enseignement et de recherche français ou étrangers, des laboratoires publics ou privés. 


\section{Deux frontières}

\section{aux destins croisés?}

Étude interdisciplinaire et comparative des délimitations territoriales entre la France et la Suisse, entre la Bourgogne et la Franche-Comté ( $x \mathrm{I}^{\mathrm{e}}$-xxI ${ }^{\mathrm{e}}$ siècle)

sous la direction de Benjamin CASTETS FONTAINE, Maxime $\mathrm{KACl}$, Jérôme LOISEAU et Alexandre MOINE 
Illustration de couverture :

(C) Jacky Frossard (Université de Franche-Comté - UFR SLHS) 


\title{
DEUX FRONTIËRES AUX DESTINS CROISÉS?
}

\author{
ÉTUDE INTERDISCIPLINAIRE \\ ET COMPARATIVE DES DÉLIMITATIONS \\ TERRITORIALES ENTRE LA FRANCE \\ ET LA SUISSE, ENTRE LA BOURGOGNE \\ ET LA FRANCHE-COMTÉ \\ (XIV ${ }^{\mathrm{e}}$-XXI ${ }^{\mathrm{e}}$ SIẼCLE)
}




\section{PRESSES UNIVERSITAIRES DE FRANCHE-COMTÉ $N^{\circ} 1448$}

\section{Collection « LES CAHIERS DE LA MSHE LEDOUX " dirigée par Philippe Barral \\ $\mathrm{n}^{\circ} 36$}

Série « Dynamiques territoriales »

Responsable : Philippe Barral

$n^{\circ} 12$

MSHE Claude Nicolas Ledoux, USR 3124

30-32, rue Mégevand

25030 Besançon cedex

(C) PRESSES UNIVERSITAIRES DE FRANCHE-COMTÉ - 2019

Mise en page et suivi éditorial : Marie Gillet 


\title{
DEUX FRONTIÈRES AUX DESTINS CROISÉS?
}

\section{ÉTUDE INTERDISCIPLINAIRE ET COMPARATIVE DES DÉLIMITATIONS TERRITORIALES ENTRE LA FRANCE ET LA SUISSE, ENTRE LA BOURGOGNE ET LA FRANCHE-COMTÉ (XIV ${ }^{\mathrm{e}}$-XXI ${ }^{\mathrm{e}}$ SIÈCLE)}

\author{
sous la direction de \\ Benjamin Castets Fontaine, Maxime Kaci, \\ Jérôme Loiseau et Alexandre Moine
}

Ouvrage publié avec le concours du Centre Lucien Febvre, de la Maison des sciences de l'homme et de l'environnement Claude Nicolas Ledoux et de la Fédération des MSH de Besançon et de Dijon 


\title{
Réseaux agronomiques et échanges transfrontaliers franco-suisses au XIX ${ }^{\mathrm{e}}$ siècle
}

\author{
Fabien KNITTEL
}

Les mondes ruraux sont des réalités très complexes qui dépassent, bien sûr, les seuls aspects liés à l'agriculture, eux-mêmes d'ailleurs, techniquement et socialement complexes. C'est pourquoi réfléchir à l'articulation entre la notion de frontière, elle aussi ambiguë et compliquée, et ces réalités rurales mouvantes est un exercice difficile (Moriceau, 2002). Vu depuis les mondes ruraux du XIX ${ }^{e}$ siècle, les frontières correspondent à des limites fluctuantes ${ }^{1}$. Toutefois, elles sont plus que des limites et le cas des mondes ruraux est un bel exemple d'enchevêtrement de délimitations. Les circulations et les mobilités «ne peuvent être exclusivement comprises dans une acception spatiale» (Hilaire-Pérez, 2015, p. 27). L'étude des échanges agronomiques transfrontaliers franco-suisses en est une illustration.

Le $\mathrm{XIX}^{\mathrm{e}}$ siècle correspond, en grande partie, à une phase d'autonomisation de la discipline agronomique, avec la fondation et la pérennisation d'institutions comme les sociétés d'agriculture, les fermes modèles ou exemplaires et les établissements d'enseignement agricole ou agronomique (Denis, 2003). Cette émergence de l'agronomie comme discipline savante/scientifique s'accompagne d'importants échanges en tous sens qui caractérisent cette phase de transition vers une agriculture plus productiviste. Ainsi, les transferts culturels et les réseaux d'échanges d'idées engendrentils l'émergence d'expériences agricoles et éducatives originales, et des croisements incessants entre les diverses cultures scientifiques à l'échelle européenne. Cette dynamique de réseau participe donc de la structuration progressive de la science agronomique (Knittel, 2010). Au sein de ces réseaux, les échanges franco-suisses occupent

1. Dans le Trésor de la langue française (TLF), les deux définitions de "frontière " sont les suivantes : "limite qui, naturellement, détermine l'étendue d'un territoire ou qui, par convention, sépare deux États "; et au sens figuré, "limite, point de séparation entre deux choses différentes ou opposées » [En ligne], URL : <http://stella.atilf.fr/Dendien/scripts/tlfiv5/visusel.exe?11;s=2373086355;r=1;nat=;sol $=0 ;>$ [consulté le 22 janvier 2016]. L'article " frontière » dans Les mots de la géographie indique : "par extension, limite séparant deux zones, deux régions, ou même deux entités plus ou moins abstraites...» (Brunet, 1993, p. 227.) 
une place essentielle du fait, en partie, du rôle clé joué par l'agronome suisse Philipp Emmanuel von Fellenberg (1771-1844)2.

À travers l'exemple privilégié des circulations des idées agronomiques entre la France et la Suisse, l'objectif est de questionner l'existence, ou non, d'un " effet frontière* ${ }^{\star}$ dans le cadre de ce cas particulier de circulations des savoirs : existe-t-il un " effet frontière* » influant sur la circulation des savoirs agronomiques franco-suisse au XIX ${ }^{e}$ siècle? Afin de répondre à cette question, nous porterons, tout d'abord, notre intérêt sur les voyages d'étude d'agronomes français en Suisse. Ensuite, à partir de l'exemple du lait, nous chercherons à savoir s'il existe une agronomie transfrontalière franco-suisse au XIX ${ }^{\mathrm{e}}$ siècle. Enfin toujours à partir de cet exemple, nous identifierons une frontière symbolique, celle du genre au sein des mondes ruraux.

\section{Voyages d'étude agronomique en Suisse}

Mathieu de Dombasle (1777-1843), l'un des premiers fondateurs de l'enseignement agricole en France (à Roville-devant-Bayon, département de la Meurthe) durant les années 1820 et artisan de l'affirmation de l'agronomie comme discipline à part entière, a été fortement attiré par la pensée d'outre-Manche. Mais les influences germaniques ont aussi laissé une empreinte forte sur sa réflexion (Knittel, 2010). Son action représente une réalité complexe, faite de croisements multiples et de transferts culturels et scientifiques imbriqués entre la France, l'Angleterre, l'Allemagne et la Suisse (on pourrait même ajouter l'Italie mais dans une moindre mesure).

Mathieu de Dombasle s'intéresse à des expériences d'enseignement agricole déjà menées en Allemagne et en Suisse. Pour donner quelques exemples, l'école d'agriculture de Tharandt, en Saxe, est fondée en 1811, tandis que l'institut agronomique de Hohenheim, de Stuttgart, est fondé en 1818. De son côté, à la même époque, Albrecht Thaër (1752-1828), une référence agronomique de premier ordre, a fondé en 1819, en Prusse, l'Institut royal d'agriculture de Moëglin (Frielinghaus et Dalchow, 2007). L'Institut agricole suisse de Hofwyl ${ }^{3}$ (près de Berne), fondé par Fellenberg, a aussi intéressé Mathieu de Dombasle.

Fellenberg acquiert en 1799 la ferme Wylhof qui devient rapidement une exploitation agricole de référence pour les agronomes européens, où il fonde, entre autres, un institut agricole et une école pour les enfants pauvres (Crud, 1816, p. 11-12). Durant les années 1810-1820, Fellenberg, dont la renommée a largement dépassé les Alpes suisses, entretient une correspondance avec Antoine Bertier, l'associé de Mathieu de Dombasle lors de la création de la ferme exemplaire de Roville-devant-Bayon ${ }^{4}$. L'agronome suisse diffuse aussi les résultats de son exploitation agricole et ses préceptes pédagogiques dans des Feuillets agricoles distribués dans toute l'Europe. Les fêtes agricoles qu'il organise en 1807 et 1810 ont d'ailleurs beaucoup de succès auprès des agronomes européens.

2. Pour quelques éléments de synthèse biographique, voir Wittwer Hesse (2002) et l'article « Fellenberg » dans le Dictionnaire historique de la Suisse : <http://www.hls-dhs-dss.ch/textes/f/F9019.php> [consulté le 20 janvier 2016].

3. Ou Hoffwyl.

4. Archives départementales de Meurthe-et-Moselle, Nancy, 7 M 37, correspondance Bertier-Fellenberg. 
Fellenberg a chargé le professeur d'agriculture Wehrli de former des enfants pauvres à devenir des valets de ferme. De son côté, en 1824-1825, Mathieu de Dombasle envisage aussi la création d'une école pour enfants pauvres pour former des ouvriers agricoles. C'est pourquoi il décide d'envoyer un jeune élève-collaborateur, Jean-Claude Fawtier, en voyage d'étude à Hofwyl. Il y séjourne deux fois six mois en 1824 et 1825. Durant ses voyages, il écrit régulièrement à Mathieu de Dombasle pour l'informer sur le fonctionnement de l'école suisse ; quatre lettres sont publiées dans les Annales agricoles de Roville, le périodique professionnel publié par Mathieu de Dombasle entre 1824 et 1832 (Dombasle, 1826, p. 199-233). Dans ses lettres, Fawtier dresse un tableau nuancé de la situation de l'école de Hofwyl et présente les enseignements du professeur d'agriculture Wehrli comme satisfaisants (Knittel, 2009, p. 375). Il le qualifie même de captivant. L'établissement d'Hofwyl est imité dans toute l'Europe, de l'Irlande à l'Autriche. Cependant, Fawtier, dans ses lettres, émet une grande réserve quant à la rentabilité économique de l'institution. Il critique l'équilibre des comptes et met en cause le fait que, par son travail, un élève couvre les dépenses qu'il occasionne. Il met donc en garde Mathieu de Dombasle en évoquant la possibilité d'un déficit financier si une telle école était ouverte à Roville. De son côté, Crud affirme que les établissements agricoles de Hofwyl, gérés par Fellenberg, sont dans une situation économique florissante (Crud, 1816, p. 6). Mais il s'agit de constats effectués pour les années 1810 à 1814 . Dix ans plus tard la situation a pu devenir moins bonne. Aussi, après l'enquête menée en Suisse par son élève, Mathieu de Dombasle, prudent, choisit-il de ne pas ouvrir le même type d'école qu'à Hofwyl, alors que l'article 17 des statuts de l'institut agricole de Roville, intégré au bail de l'exploitation, envisageait une telle création (Knittel, 2009, p. 375). Dans les faits cependant, des jeunes garçons démunis sont formés, à Roville, de façon informelle, aux fonctions de valets de ferme.

Un autre agronome lorrain, Louis Poirot de Valcourt (1771-1855), s'est rendu à Hofwyl auprès de Fellenberg, durant l'été 1833 (Knittel, 2015). Durant son séjour, Poirot de Valcourt propose à l'agronome suisse « de lui installer une de ses charrues, modèle écossais, sur un avant-train avec les leviers à la Grangé ${ }^{\prime}$ (Poirot de Valcourt, 1841, p. 122). Fellenberg refuse mais uniquement pour des questions pédagogiques et non techniques : ce procédé rendrait le travail de labour trop aisé pour des jeunes gens qui apprennent à manier la charrue. Par la suite, les deux agronomes poursuivent leurs échanges de manière épistolaire.

\section{Une agronomie transfrontalière : circulation des savoirs agronomiques sur le lait entre Franche-Comté et Suisse}

L'immigration des fromagers suisses dans la France de l'Est est un fait majeur à partir du dernier tiers du XIX ${ }^{\mathrm{e}}$ siècle (Kronenberger, 2014, p. 94-174). Ce sont eux qui importent les techniques de fabrication du fromage d'emmental, inconnu en France auparavant (Delfosse, 1994, p. 133-144 ; Lachiver, 2006, p. 523). En Suisse, le principe $\mathrm{du}$ " tour » est pratiqué comme en Franche-Comté : le fruitier se rend chez chacun des associés de la coopérative à tour de rôle pour fabriquer le fromage (on l'appelle aussi fruitières à petit carnet). D'après Charles Lullin, auteur, en 1811, Des associations rurales pour la fabrication du lait (p. 31), celui qui accueille le fruitier doit fournir le

5. Sur le « système Grangé », voir Knittel (2007). 
bois utilisé pour chauffer le lait ainsi qu'un essuie-main. L'outillage, terme pris ici dans son sens le plus large, n'est pas entièrement exclusif au fruitier qui utilise aussi dans le cadre de sa pratique quotidienne des « ustensiles » fournis par les associés de la fruitière. Les techniques de fabrications ne sont donc pas un savoir-faire totalement propre au fruitier mais des gestes connus dans les campagnes et que beaucoup de paysans, notamment les éleveurs laitiers du Jura, de part et d'autre de la frontière, connaissent et pratiques plus ou moins régulièrement.

Dans les textes techniques des agronomes, tant suisses que français, le souci de la propreté des ustensiles utilisés est constant et cela fait partie des recommandations les plus fréquentes (par ex. Lullin, 1811 ; Poirot de Valcourt, 1841). Le fruitier se voit recommander de laver les ustensiles à la fin de chaque fabrication, soit une fois par jour, puis de laver la pièce où cette fabrication s'est déroulée afin de faciliter le séchage des baquets qui contiennent le lait. Lullin précise qu' « il ne faut épargner aucune peine et aucun soin pour entretenir dans le laitier une propreté absolue » (Lullin, 1811, p. 80-81). Cette insistance sur la propreté des lieux de fabrication se retrouve, trente ans plus tard, chez Poirot de Valcourt qui explique, par exemple, que la « fille de ferme " doit utiliser " un baquet et un balai pour nettoyer l'étable qui est lavée et sablée deux fois par jour » (Poirot de Valcourt, 1841, p. 226). C’est une préoccupation qui, au cours de la seconde moitié du XIX ${ }^{e}$ siècle, rencontre un contexte de plus en plus favorable avec l'essor de l'hygiénisme (Bruegel et Stanziani, 2004, p. 7-16). Ce souci de la propreté est essentiel pour la fabrication des fromages, notamment pour le gruyère et l'emmental pour lesquels le lait doit être abondant et propre (Delfosse, 1994, p. 139 et p. 141).

Pour fabriquer le beurre, en Suisse dans les années 1810-1820, Lullin explique que l'on utilise une sérenne, outil qui se rapproche de la baratte si on suit sa description. Elle s'utilise, nous dit-il, en effectuant des gestes « dou[x] et réglé[s] » (Lullin, 1811, p. 83), c'est-à-dire une cadence lente mais uniforme qui annonce un travail fastidieux fondé sur la répétition sur une durée assez longue du même geste du bras. Une fois l'opération terminée, le beurre est retiré à la main puis pétri par le fruitier afin de retirer tout le lait résiduel.

Pour éviter la fraude, l'examen visuel et olfactif du lait est l'une des étapes clés du travail des fruitiers. Lullin indique que le fruitier expérimenté reconnaît facilement à la vue ou au goût un lait de mauvaise qualité. Mais cela n'est pas suffisant, il recommande alors l'usage d'un instrument de mesure : ici « un pèse-liqueur gradué » (ibid., p. 72). Toutefois, cette pratique de la mesure est encore limitée au début des années $1810 \mathrm{chez}$ les fromagers suisses. L'auteur remarque que "l'éprouvette [ou galactomètre] n'indique point d'une manière absolue la richesse du lait... ( (ibid., p. 73-74). Plus tard, durant les années 1830 et 1840 , le lactomètre, "tube en verre, muni d'un pied, de la contenance d'un peu plus de 2 décilitres, divisé en 100 parties » (Poirot de Valcourt, 1841, p. 201), devient un instrument de mesure de plus en plus important pour l'industrie laitière et les exploitants agricoles qui transforment le beurre à la ferme. Poirot de Valcourt dans ses Mémoires sur l'agriculture attribue l'invention du lactomètre à Fellenberg. Avec le lactomètre, les crémiers, les fromagers, les fruitiers francs-comtois peuvent ainsi vérifier la qualité du lait qu'on leur livre tandis que, de son côté, l'éleveur peut utiliser les mesures du lactomètre comme un indicateur pour améliorer ses pratiques d'élevage. Les pratiques de mesure, lancées pour lutter contre 
la fraude, font donc l'objet d'une intense circulation des savoirs entre la France et la Suisse.

Or, cette circulation des savoirs n'est pas toujours aussi fluide. Ainsi, si Charles J. Martin, premier directeur de l'École nationale d'industrie laitière de Mamirolle (non loin de Besançon) fondée en 1888, admet que le savoir-faire des fromagers suisses est réputé, il vante surtout le savoir-faire des fruitiers francs-comtois (Martin, 1898, p. 5-9). C'est une sorte de chauvinisme économique qui se comprend bien dans un contexte de forte concurrence transfrontalière avec un marché du lait en crise depuis le début des années 1880 (Delbaere, 2010, p. 84). Les fromagers suisses qui immigrent en France, principalement en Franche-Comté au milieu du XIX ${ }^{\mathrm{e}}$ siècle, ne possèdent souvent que leur savoir-faire. Ce dernier est acquis par apprentissage dans les fruitières ou alors dans des écoles spécialisées fondées bien plus tôt qu’en France, comme l'école laitière de Rütti ouverte en 1860, près de Berne, par exemple (Delfosse, 1994, p. 134). Parfois les fromagers suisses refusent d'avoir des élèves. Cependant ces fromagers suisses importent avec eux une certaine émulation technique et favorisent le perfectionnement des techniques de fabrication du gruyère et de l'emmental (ibid., p. 138), avant les bouleversements scientifiques et techniques introduits par Pasteur et les pastoriens après les années 1870 (Delbaere, 2007, p. 118).

Mais au-delà de la frontière franco-suisse, la fabrication du beurre génère une autre frontière, celle de la répartition sexuée des tâches au sein de l'exploitation agricole.

\section{La frontière du genre dans les exploitations laitières}

L'association, dans les sociétés rurales traditionnelles, entre la culture des céréales et les hommes, d'une part, et entre la fabrication du lait et du beurre et les femmes, d'autre part, est bien connue (Cocaud, 2004, p. 101-112). Les rapports sociaux inégaux de sexe au sein du monde rural transparaissent de manière forte lorsque l'on étudie la répartition sexuée des tâches au moment de la fabrication du beurre et, plus rarement, du fromage. Répartition genrée du travail qui ne se modifie guère durant le dernier tiers du XIX ${ }^{\mathrm{e}}$ siècle avec l'industrialisation de la transformation du lait.

La fabrication du beurre est une tâche féminine assurée au sein de l'exploitation familiale. Le principal lieu de transformation du lait entre 1850 et 1870 reste l'exploitation agricole où la "fermière » utilise souvent une écrémeuse manuelle (Delbaere, 2007, p. 137 et p. 152). La connaissance de ces pratiques quotidiennes n'est pas toujours très fine car elles se déroulent au sein de l'espace domestique de l'exploitation qui correspond à la sphère la moins documentée par les historiens des mondes ruraux, donc la moins connue (Cocaud, 2004, p. 103). Toutefois, même si nos connaissances dans ce domaine restent encore parcellaires, il existe un certain nombre d'informations à notre disposition. Dans la montagne jurassienne, Jean-Luc Mayaud a montré que les épouses de chef d'exploitation laitière sont chargées de s'occuper du bétail, surtout lorsque le mari exerce, en plus de son travail agricole, une activité artisanale (Mayaud, 1999). Ce sont les femmes qui assurent alors la traite et la gestion du lait ainsi que sa transformation lorsqu'elle s'effectue sur place comme c'est encore très souvent le cas au milieu du XIX ${ }^{\mathrm{e}}$ siècle, notamment dans les "montagnes à lait » que sont les Vosges ou le Jura (Sainclivier, 2005). La situation "genrée » identifiée par Jean-Luc Mayaud du côté français du Jura se retrouve, peu ou prou, sur le versant suisse. Il s'agit d'une répartition sexuée traditionnelle du travail. Ici, la frontière du 
genre est transfrontalière. Les commentaires des agronomes, du côté français comme du côté suisse, sont laconiques (voire anecdotiques) sur ce point car leur intérêt se porte davantage sur les pratiques innovantes, qui concernent principalement des hommes, que sur les tâches ordinaires, qui concernent cette fois-ci aussi les femmes (par ex. Lullin, 1811).

Si le travail féminin tend à diminuer pour les travaux en plein champ durant le $\mathrm{XIX}^{\mathrm{e}}$ siècle, bien qu'il existe d'importantes nuances régionales, les femmes restent chargées principalement de l'entretien du foyer, de la basse-cour et de la laiterie, en France comme en Suisse (Knittel et Raggi, 2013, p. 19). On remarque donc que la division sexuée des diverses tâches agricoles est fondée sur une complémentarité de celles-ci, une manière d'assigner à chacun et chacune sa place dans l'ordre social rural (Cocaud, 2004, p. 107). À cette division genrée s'ajoute une division par âge. Les jeunes garçons participent aussi à la traite des vaches et à la fabrication du beurre, mais contrairement aux filles et femmes qui s'occupent de ces tâches durant toute leur vie, les hommes devenus adultes n'y participent plus (Bourdon, 2004, p. 131). Ils se consacrent aux tâches traditionnellement assignées à leur sexe, sauf lorsqu'ils décident de devenir fruitiers. Mais à ce moment-là ils sortent de l'exploitation familiale et participent à l'industrialisation de la transformation du lait.

Les femmes assurent donc, au sein de l'exploitation agricole familiale, l'ensemble du processus de fabrication depuis la traite jusqu'à la transformation en beurre et, parfois aussi, de certains fromages. Elles s'occupent aussi de la vente des produits laitiers en ville : elles sont alors appelées laitières (Delbaere, 2007, p. 130 ; Bourdon, 2004, p. 113-114). L'image de la laitière qui porte son lait pour le vendre en ville devient une figure stéréotypée de la peinture ou de la sculpture populaire, se transformant en véritable poncif dans la seconde moitié du XIX ${ }^{\mathrm{e}}$ siècle, parallèlement à l'augmentation de la production laitière (Bourdon, 2004, p. 114-115 et p. 121-127).

Or, une fois sortie de la ferme, et mis à part la vente des produits laitiers, ce sont les hommes qui fabriquent les fromages au sein des fruitières et, parfois, même le beurre. La production domestique, fermière pourrait-on dire, est le domaine réservé en quelque sorte des femmes tandis que la transformation industrielle des produits du lait est assurée par les hommes, qui bénéficient le plus souvent d'un apprentissage, lequel est scolarisé à partir de la fin des années 1880 avec la création des écoles de laiterie (Charmasson et al., 1999, p. 47 et 55 ; Knittel, 2014, p. 119-132).

D'effet frontière ${ }^{\star}$ il semble donc y avoir mais souvent en proportion assez faible. Si l'exemple du lait est significatif des circulations transfrontalières des savoirs agronomiques, l'absinthe est un autre exemple local d'un effet frontière ${ }^{\star}$ franco-suisse assez faible dans les domaines agricoles et ruraux. C'est davantage un marqueur de la frontière entre l'urbain et le rural. Les plantes, c'est-à-dire la grande absinthe et la petite absinthe qui entrent, l'une et l'autre, dans la fabrication de l'alcool d'absinthe, du côté français, sont cultivées et récoltées dans les espaces ruraux du Jura (au-dessus de 1000 mètres d'altitude) mais n'y sont pas distillées. Les distilleries se trouvent principalement en ville, comme, par exemple, la distillerie Pernod à Pontarlier, depuis le début du XIX ${ }^{\mathrm{e}}$ siècle (Delahaye, 2005 et 2010). En Suisse, en revanche, davantage de distilleries se trouvent au cour de l'espace rural du Val-de-Travers (Hertz, 2011 ; voir aussi Delachaux, 1997). 
Les rapports et échanges entre agronomes relèvent de la circulation des savoirs à l'échelle européenne à travers des réseaux relativement pérennes et croisés qui ne tiennent que très peu compte des frontières étatiques (Knittel, 2009). À l'échelle des pratiques quotidiennes, la répartition sexuée des tâches dans le cadre de la transformation du lait, se retrouve de part et d'autre de la frontière franco-suisse : la frontière de genre est ici transfrontalière et si effet frontière* il y a, c'est un effet déterritorialisé qui est caractéristique des sociétés agraires traditionnelles, une frontière de genre. Le seul effet frontière* notable, mais souvent très atténué, peut être lié aux langues différentes parlées par les agronomes. Mais la plupart d'entre eux sont polyglottes ou maîtrisent en partie la langue de leur interlocuteur (ibid.). Cela permet d'ailleurs une circulation rapide des savoirs et des innovations à l'échelle européenne ainsi que des polémiques nées des propositions scientifiques et/ou techniques des uns ou des autres (Diederen, 2003).

\section{Bibliographie}

Bourdon Jean-Paul, 2004 , "La laitière et le citadin normand ( $\mathrm{XIX}^{\mathrm{e}}-\mathrm{XX}^{\mathrm{e}}$ siècles) ", Enquêtes rurales, $\mathrm{n}^{\circ} 10$, p. 113-135.

Brunet Roger (dir.), 1993, Les mots de la géographie. Dictionnaire critique, Paris, La Documentation française.

Bruegel Martin et Stanziani Alessandro, 2004, "Pour une histoire de la sécurité alimentaire », Revue d'histoire moderne et contemporaine, vol. 5, $\mathrm{n}^{\circ} 3$, p. 7-16.

Charmasson Thérèse, Duvigneau Michel, Lelorrain Anne-Marie, Le Naou Henri, 1999, L'enseignement agricole, 150 ans d'histoire, Dijon, Éducagri.

Cocaud Martine, 2004, "Les femmes de la campagne à l'époque contemporaine. Essai de bibliographie », Enquêtes rurales, n 10, p. 101-112.

Crud Benjamin, 1816, Notice sur les établissements de Hofwyl, Paris/Genève, J. J. Paschoud.

Delachaux Pierre-André, 1997, «L'absinthe au Val-de-Travers : recherches sur ses origines ", Revue historique neuchâteloise, $\mathrm{n}^{\circ}$ 1, p. 3-22.

Delahaye Marie-Claude, 2010, L'absinthe, de Pontarlier au Val-de-Travers : d'hier à aujourd'hui, Auvers-sur-Oise, Musée de l'absinthe.

- 2005, Pernod: 200 ans d'entreprise, Auvers-sur-Oise, Musée de l'absinthe.

- 2001, L'absinthe. Son histoire, Auvers-sur-Oise, Musée de l'absinthe.

Delbaere Nicolas, 2010 (janvier-juin) « L'État et la formation professionnelle laitière de 1880 à 1914 », Cahiers Jaurès, n 195-196, p. 81-102.

- 2007, L'économie laitière dans le Nord-Pas-de-Calais, de l'âge rural à l'âge des marques, thèse de doctorat d'histoire, Lille, Université de Lille 3.

Delfosse Claire, 1994, «Le savoir-faire des fromagers suisses de la France de l'Est (1850-1950) », Études rurales, nº 135-136, p. 133-144. 
Denis Gilles, 2003 [1999], "Agronomie », in Lecourt Dominique (dir.), Dictionnaire d'histoire et philosophie des sciences, Paris, Presses universitaires de France, p. 24-29.

Diederen Peter, Van Meijl Hans, Wolters Arjan et Bijak Katarzyna, 2003, « Innovation adoption in agriculture: innovators, early adopters and laggards ", Cahiers Économie et Sociologie rurale, n 67, p. 29-50.

Dombasle Mathieu (de), 1826, "Lettres sur l'école d'industrie de Hofwyl, écrites à Mathieu de Dombasle, par M. Fawtier, élève à Roville », Annales agricoles de Roville, $\mathrm{n}^{\circ}$ 3, p. 199-233.

Frielinghaus Martin et Dalchow Claus, 2007, « Thaër, 200 years at Möglin (Germany) ", in Robin Paul, Aeschlimann Jean-Paul, Feller Christian (dir.), Histoire et agronomie : entre rupture et durée, Paris, Institut de recherches pour le développement, p. 259-267.

Hertz Ellen, 2011, Vive le mythe? Vive l'absinthe! Une recherche de terrain au Valde-Travers, mini-mémoire pour le séminaire "Le patrimoine immatériel à Neuchâtel : entre découverte et invention », Neuchâtel, Université de Neuchâtel.

Hilaire-PÉrez Liliane, 2015, "Savoirs et mobilités à l'échelle du monde : un paradigme au prisme de la recherche collective ", in Gonzalez Bernaldo Pilar et Hilaire-PÉrez Liliane (dir.), Les savoirs-mondes, Rennes, Presses universitaires de Rennes, p. 17-30.

Knittel Fabien, 2015, De la bibliothèque aux champs. Le travail d'agronome de Louis Poirot de Valcourt (1771-1855), Nancy, Presse universitaires de Nancy/ Éditions universitaires de Lorraine.

- 2014, «L'"éducation" des fruitiers et des laitières de Franche-Comté au XIX ${ }^{\mathrm{e}}$ siècle : entre initiation domestique, apprentissage professionnel et transmission scolaire », Les Études sociales, n 159 , p. 119-132.

- 2010, "L'Europe agronomique de C. J. A. Mathieu de Dombasle », Revue d'histoire moderne et contemporaine, vol. 57, $\mathrm{n}^{\circ} 1, \mathrm{p} .119-138$.

- 2009, Agronomie et innovation. Le cas Mathieu de Dombasle (1777-1843), Nancy, Presses universitaires de Nancy.

— 2007, « La charrue Grangé ou le parcours atypique d'un valet de charrue, vers 1830 en Lorraine », in Bourrigaud René et Sigaut François (dir.), Nous labourons, Nantes, Centre d'histoire du travail, p. 331-339.

Knittel Fabien et Raggi Pascal, 2013, «Des techniques au genre et retour », in KNITTEL Fabien et RAGGi Pascal (dir.), Genre et techniques (XIX $\mathrm{X}^{e} \mathrm{X} \mathrm{X}^{e}$ siècles), Rennes, Presses universitaires de Rennes, p. 11-21.

Kronenberger Stéphane, 2014, Des temps de paix aux temps de guerre: les parcours des travailleurs étrangers de l'est et du sud-est de la France (1871-1918), thèse de doctorat en histoire, Nice, Université de Nice-Sophia Antipolis.

LaCHIVer Marcel, 2006 [1997], Dictionnaire du monde rural : les mots du passé, Paris, Fayard. 
Lullin, Charles, 1811, Des associations rurales pour la fabrication du lait, connues en Suisse sous le nom de fruitières, Paris/Genève, J. J. Paschoud.

Martin Charles J., 1898, Les fruitières du Doubs, Besançon, Impr. du progrès.

Mayaud Jean-Luc, 1999, La petite exploitation triomphante. France, XIX ${ }^{e}$ siècle, Paris, Belin.

Moriceau Jean-Marc, 2002, Terres mouvantes, Les campagnes françaises du féodalisme à la mondialisation, XII ${ }^{e}-X I X^{e}$ siècle, Paris, Fayard.

Nourrisson Didier, 1990, Le buveur du XIX ${ }^{e}$ siècle, Paris, Albin Michel.

Poirot de Valcourt Louis, 1841, Mémoires sur l'agriculture, Paris, Bouchar-Huzard.

SAINCLIVIER Jacqueline, 2005, «Une histoire des agricultrices aux $X I X^{e}-X X^{e}$ siècles est-elle possible en France? Acquis et perspectives ", in Vivier Nadine (dir.), Ruralité française et britannique. Approches comparées, Rennes, Presses universitaires de Rennes, p. 117-128.

Wittwer Hesse Denise, 2002, Die Familie von Fellenberg und die Schulen von Hofwyl, Bern, Historischer Verein des Kantons Bern. 


\section{Table des matières}

\section{Introduction}

Benjamin CAstets Fontaine et Maxime KaCi

Première partie. De la violence frontalière : des territoires à risques?

Introduction

Oscar Mazzoleni

1 Microhistoire d'une triple frontière : les «terres de surséance » entre duché de Bourgogne, Franche-Comté et Champagne sous Charles VII et Louis XI (1435-1477)

Léonard DAUPHANT

2 Une modernité divergente : les deux Bourgognes et la monarchie française $\left(\mathrm{XVI}^{\mathrm{e}}-\mathrm{XVII}^{\mathrm{e}}\right.$ siècle $)$

Jérôme LOISEAU

3 Recompositions frontalières en révolution : quand les affrontements politiques transcendent les appartenances provinciales et nationales (1789-1798)

Maxime KACI

4 La vache et le territoire

Dominique JACQUES-Jouvenot

5 De la frontière haineuse à la frontière aimante : des espaces en question Laurent Tissot

Deuxième partie. La différenciation territoriale, une opportunité?

Introduction

Julien Chevillard et Stéphane Kronenberger. 
6 Des identités éclatées : l'Arc jurassien entre Confédération, France et Saint Empire (début XVI ${ }^{\mathrm{e}}$-fin XVII ${ }^{\mathrm{e}}$ siècle)

Bertrand ForCLAZ

7 Chronique d'une disparition? Une géohistoire de la « frontière » Bourgogne/ Franche-Comté (1790-2016)

Robert CHAPUIS

8 Réseaux agronomiques et échanges transfrontaliers franco-suisses au XIX ${ }^{\mathrm{e}}$ siècle

Fabien KnITTEL

9 Les mobilités professionnelles transfrontalières : dynamique générationnelle et stratification sociale

Julien CHEvillard

Troisième partie. Contrôler, construire et aménager les territoires frontaliers : des préoccupations contrastées

Introduction

Annie Bleton-Ruget

10 Bâtir un espace économique par-delà les ressorts. Le duc Philippe le Hardi, la réglementation du sel et la question des terres d'outre-Saône

Michelle BubeniceK.

11 La frontière franco-suisse de la paix à la guerre (1871-1918)

Stéphane KRONENBERgER

12 Faire cause commune au-delà de la frontière ? Le syndicalisme transjurassien en échec

Dominique Andolfatto

13 Les effets frontières des politiques publiques d'aménagement du territoire. L'exemple du pays de la Bresse bourguignonne et du pays lédonien Annie Bleton-Ruget

14 Une frontière internationale comme catalyseur de dynamiques? Le cas ambigu de l'Arc jurassien franco-suisse

Alexandre MoIne.

\section{Conclusion}

Jérôme LoISEAu et Alxandre MoInE

Annexes.

Sigles et acronymes.

Lexique

Index géographique

Index des noms propres 


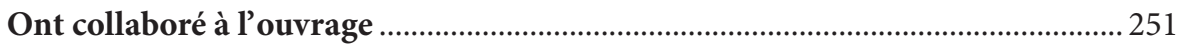

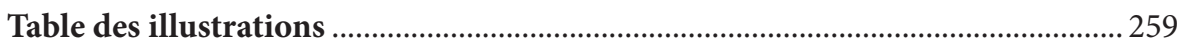


Imprimerie Messages

111, rue Nicolas Vauquelin - 31100 Toulouse

Dépôt légal : deuxième trimestre 2019

Imprimé en France 
Fruit d'échanges interdisciplinaires, cet ouvrage interroge les liens entre délimitations territoriales et organisation des sociétés. Suivant une démarche comparative, les auteurs questionnent, depuis les débuts des États modernes au XIV siècle jusqu'à aujourd'hui, les effets induits par les évolutions de ces délimitations à partir de deux terrains d'observation contigus aux évolutions a priori opposées : les territoires entre Bourgogne et Franche-Comté où semble disparaître toute limite; ceux entre France et Suisse où s'affirme l'une des dernières frontières entre la France et un État non adhérent de l'Union européenne. Cette comparaison permet de déterminer ce qui distingue une frontière étatique de délimitations territoriales d'autres natures.

Initiée par Maxime $\mathrm{KACl}$ (maître de conférences en histoire), en collaboration avec Benjamin CASTETS FonTAINE (maître de conférences en sociologie), Jérôme LoISEAU (maître de conférences en histoire) et Alexandre MoINE (professeur de géographie), cette publication réunit seize chercheurs originaires de pays différents.
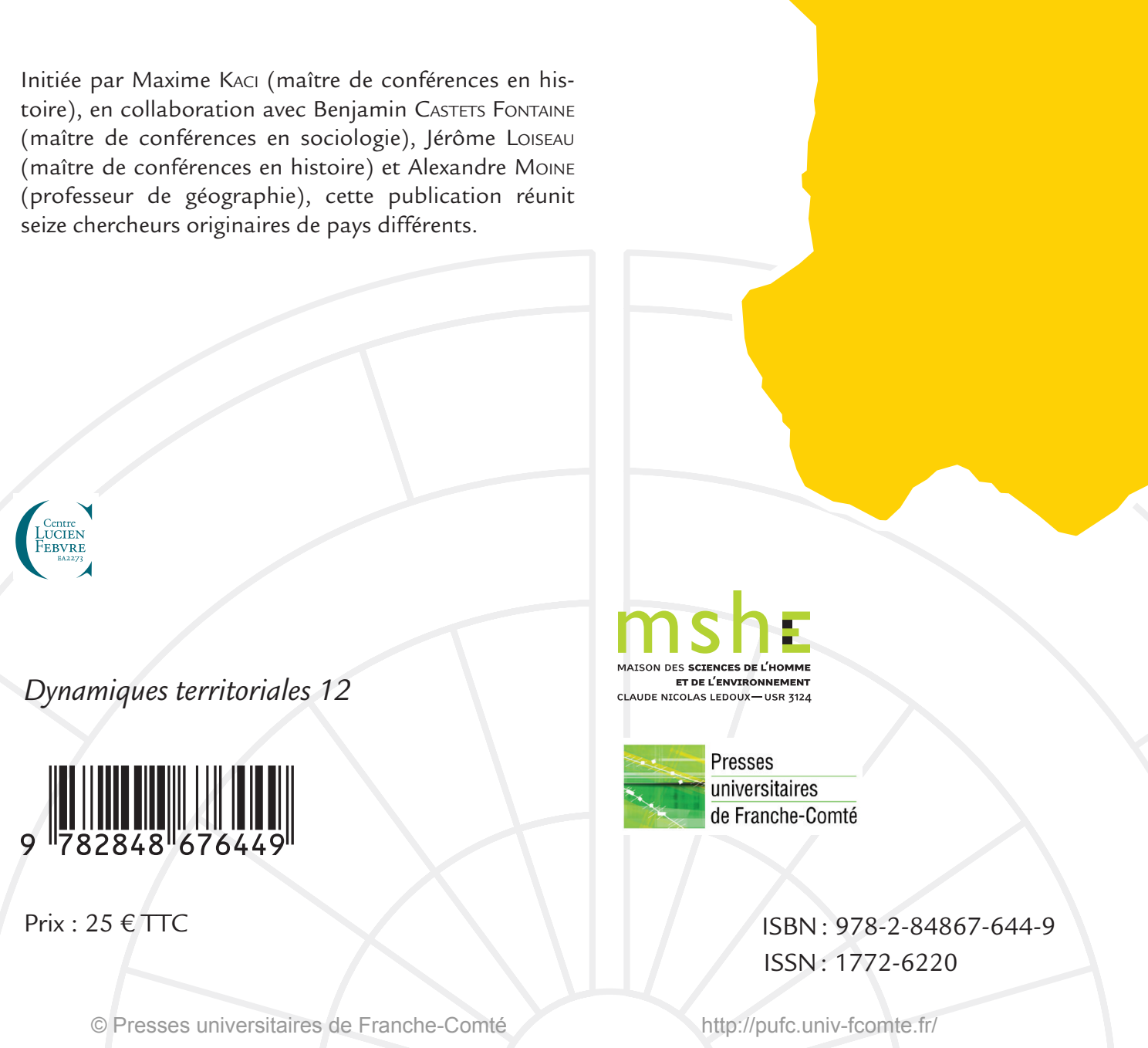

Dynamiques territoriales 12

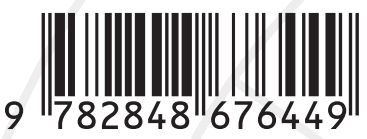

Prix : $25 € \Pi C$ 